\title{
Electronic Epidemiologic Biosurveillance in the Cameroon Military
}

\author{
Karen Saylors*, Matthew LeBreton, Essisima Foe, Aboudem Bavou Clement Martial, \\ Nancy Ortiz, Christopher Perdue and Ubald Tamoufe
}

Metabiota, San Francisco, CA, USA

\section{Objective}

Pilot and evaluate an electronic disease surveillance system in the Cameroon military and assess the capabilities of this system to fulfill reporting and early warning requirements.

\section{Introduction}

Electronic epi-biosurveillance presents an opportunity to provide real-time disease surveillance alerts from remote areas to central disease management units, to rapidly decrease reporting times for reportable diseases, and to enable appropriate response scenarios to be put in place in a timely manner.

Over the past year, with the support of GEIS and Johns Hopkins Applied Physics Lab, we have piloted an electronic disease reporting system in four sites in the Cameroon military and evaluated these surveillance efforts, to understand how such infrastructure may impact this resource-limited setting.

\section{Methods}

Over the past 11 months, we piloted an electronic reporting system in the Cameroon military, SAGES (Suite for Automated Global Epidemiological bioSurveillance), where notifiable disease data is entered daily via smart phones. Two military hospitals and two clinics were included, covering two of the three military regions in Cameroon. The daily number of suspected cases of 24 reportable diseases with epidemic potential (as defined by WHO) were compiled daily and sent to a central server. Access to incoming data was provided to central level health managers in the Ministry of Defense and the Ministry of Health. We evaluated SAGES to determine users situational awareness of alert reporting and whether the system engenders engagement from higher level military when SAGES notifies of highly contagious cases or outbreaks.

\section{Results}

End users rapidly adapted to the system despite low current levels of smartphone usage in Cameroon. Rapid access to real time data was easily obtained, including notifications of suspected cases of diseases including malaria, pneumonia, gastroenteritis, and in one site, STIs. Difficulties included the lack of existing systems of documented consultations in the hospitals, lack of knowledge of and adherence to case definitions, lack of IT infrastructure (hardware and internet service) at the central level for accessing and viewing data, and in particular the difficulty of compiling reporting from different service departments in larger hospitals which were not effectively communicating or previously documenting consultations. In the evaluation of SAGES, $73.68 \%$ responded positively that SAGES alerts, notifying of highly contagious cases or outbreaks, provoke reaction from higher level military, leading to outbreak investigation and management, as intended. Although case definition continues to be a challenge in this resource-limited setting, $94.73 \%$ of users said SAGES has improved their case reporting.

\section{Conclusions}

The pilot of this electronic epidemiological biosurveillance demonstrated that even in resource-limited environments with limited smart phone coverage, using SAGES as a platform for disease surveillance could be successful and taken to scale, assuming that consistent training and technical support is provided. However this system, which requires all cases to be compiled and submitted in a single data transfer, may be better adapted to small health structures rather than larger hospitals with multiple clinicians in multiple service departments. If SAGES is to be taken to a nationwide scale in this resource-limited region, continued technical support, advanced clinical training on case definitions, and development of a standardized operational response to alerts and outbreak characterization and management will be necessary.

\section{Keywords}

electronic surveillance; resource-limited settings; evaluation

\section{Acknowledgments}

This work was supported by the US Armed Forces Health Surveillance Center Division of GEIS Operations, with technical assistance from Johns Hopkins University Applied Physics Lab.

\section{References}

1. Lewis, SL, Feighner, BH, Loschen WA, Wojcik RA, Skora JF, Coberly JS, Blazes DL. SAGES: A Suite of Freely-Available Software Tools for Electronic Disease Surveillance in Resource-Limited Settings, PLOS One 6(5), 2011.

2. Savel, TG, Foldy $\mathrm{S}$. The role of public health informatics in enhancing public health surveillance. MMWR Surveill Summ 2012; 61: 20-24.

\footnotetext{
*Karen Saylors

E-mail: ksaylors@metabiota.com
} 